\title{
Elogio de una nueva sección a propósito de la optimización del tubo
}

\section{Praise of a new section regarding tubular optimization}

\section{RESUMEN}

Las secciones tubulares ofrecen una respuesta excelente frente a multitud de solicitaciones diversas. A pesar de esto, el uso de este tipo de secciones es relativamente reciente por su desconocimiento y su complejidad de comportamiento. Combinándolas con un relleno de hormigón, mejoran notablemente su rendimiento para dar lugar a unas secciones mixtas, en dónde ambos materiales no sólo se complementan sino que en muchos casos mejoran sus prestaciones. Este texto enumera las ventajas de este tipo de secciones, a la vez que analiza la aplicabilidad de una nueva tipología seccional que va un paso más allá de las mixtas convencionales: las tubulares mixtas aligeradas. Esta nueva tipología optimiza la resistencia seccional a la vez que rentabiliza material frente al pandeo del pilar. El artículo pretende divulgar y proponer la introducción de este tipo de secciones en las nuevas arquitecturas, desde el punto de vista de la prefabricación.

\section{SUMMARY}

Tubular sections have an excellent response under combined stress states. However, the use of this typology has been relatively recent, mainly due to the unfamiliarity in use. By filling them with concrete [composite sections] the two materials do not work only together, but they also improve their mechanical properties. This text points out the advantages of these sections and proposes the applicability of a new typology which goes beyond in structural optimization: hollow concrete-filled steel tubes. The hollowness in composite tubular sections improves their mechanical performance, and it also optimizes its behavior in case of slender columns. This paper pretends to divulge and encourage the use of these sections in new architectural designs, from the point of view of high-rise building and prefabrication.
Palabras clave: Secciones tubulares; secciones mixtas; secciones mixtas tubulares aligeradas; pilares; optimización.
Keywords: Tubular sections; composite sections; hollow concrete-filled sections; columns; optimization.

\footnotetext{
(*) ETSAB-Universitat Politècnica de Catalunya, Barcelona (España). 
1. Croquis de Violet le Duc. Fuente: http://www.brynmawr.edu

2. Puente Firth of Forth, Edinburgh.

Fuente: http://www.puentemania.com

\section{LA INTRODUCCIÓN DEL TUBO EN LA ARQUITECTURA}

La historia estructural es una historia basada en la optimización desde muchos puntos de vista. Optimización en relación a la geometría, al material y a la sección con el objetivo de ir cada vez más lejos en el camino de la eficacia estructural, y poder así resolver problemas de complejidad mayor.

Desde los inicios de la arquitectura, y fruto de los materiales de que se disponía, se apostó por una adaptación de las geometrías arquitectónicas a las prestaciones mecánicas de éstos con el fin de aprovechar al máximo sus posibilidades. Siglos después, con el estallido de la Revolución Industrial, aparecieron nuevos materiales que supusieron un avance sin precedentes en la optimización estructural, implicando a su vez también cambios a nivel seccional. Cada vez que se han producido mejoras en el rendimiento estructural, éstas han ido lógicamente acompañadas de nuevos retos arquitectónicos.

Las secciones tubulares han entrado relativamente tarde en el diseño arquitectónico; ya sea por su uso predominantemente industrial, o bien por la dificultad de ejecución de sus uniones entre barras de la estructura, éstas han sido relegadas muchas veces del repertorio seccional disponible, a pesar de sus extraordinarios atributos.

El uso de perfiles tubulares se remonta a los primeros pilares de fundición, justo en los orígenes de este material; edificios como el Crystal Palace incorporan repetidamente este tipo de secciones cerradas, y grandes pensadores como Violet le Duc predijeron su comportamiento ejemplar trabajando a compresión, con el fin de resolver problemas de una complejidad contemporánea (Figura 1). Cabe especificar que a pesar de que muchas veces se plantearon las ventajas de los tubos para edificios más bien singulares, la industria tuvo un papel determinante durante mucho tiempo, mostrándose reticente a sustituir los perfiles de edificación habituales laminados en $\mathrm{I}$ o $\mathrm{H}$.

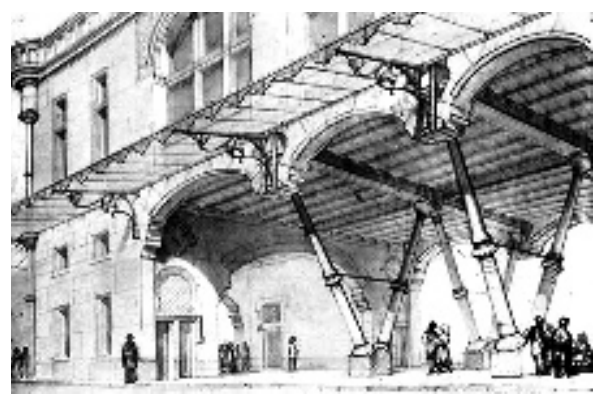

Todas estas aplicaciones iniciáticas fueron siempre hechas en fundición. El acero no se fabricó hasta mucho tiempo después, por lo que las primeras secciones tubulares respondían únicamente a solicitaciones puras de compresión.

El uso explícito de perfiles tubulares en obra civil se planteó por primera vez de forma seria hace 150 años en dos puentes de ferrocarril británicos: el Britannia Railway Bridge y el Saltash Railway Bridge. A partir de aquí y muy lentamente, fueron generalizándose estas secciones en el uso industrial, y entre los años 1882 y 1890 se construyó por primera vez la totalidad de una gran obra civil mediante secciones tubulares roblonadas: el conocido puente Firth of Forth, en la ciudad escocesa de Edinburgh (Figura 2). La obra se convirtió en referencia en el mundo de la ingeniería civil y estableció precedentes para la divulgación de un tipo de sección con la que no podían competir las secciones abiertas en $\mathrm{H}$, por sus extraordinarias prestaciones.

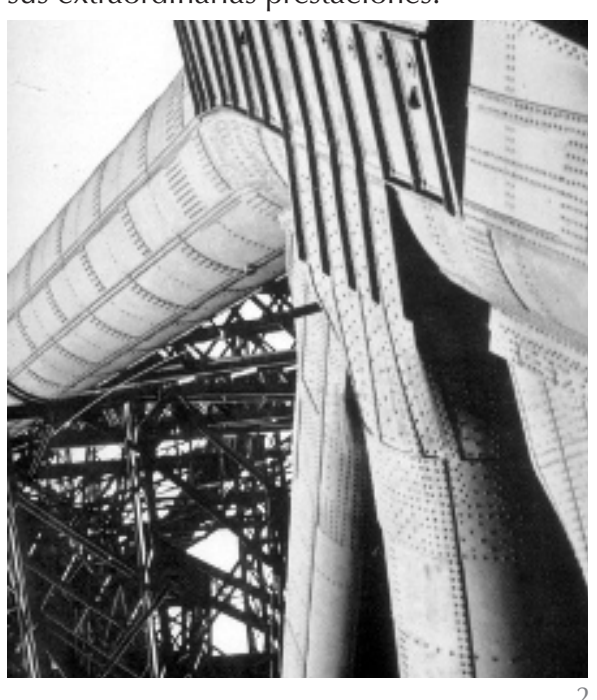

El siguiente paso -decisivo- en la aplicación de perfiles tubulares, lo llevó a cabo Alexander Graham Bell construyendo por primera vez una malla espacial de tubos metálicos para uso aeronáutico, que poco después pasó a utilizarse también en obra civil. Ésta se considera la primera malla espacial construida en la historia, pensada inicialmente a base de barras tubulares (1). En los años 40 se hizo un amplio uso de las secciones tubulares, sobre todo en estructuras espaciales; en el año 1942 Max Mangeringhausen propuso la primera malla tridimensional resuelta con tubos articulados entre sí en los nodos. Es a partir de este momento que el uso de secciones tubulares para estructuras ligeras se generaliza ampliamente.

La aparición del CIDECT (Organización de Fabricantes de Perfiles Tubulares) ha hecho 
posible realizar un proceso de divulgación enorme de las ventajas de este tipo de perfiles, con lo que se han implantado con bastante éxito en las arquitecturas de la segunda mitad del siglo XX. Un factor decisivo en esta expansión ha sido la incorporación del hormigón en su interior y la conversión de unas estructuras estrictamente metálicas en mixtas, de rendimiento notablemente superior.

\section{EL CONCEPTO DE SECCIÓN MIXTA}

Del mismo modo que en el caso de las secciones tubulares, el concepto de sección mixta en columnas, en las que pudiera considerarse un trabajo conjunto de hormigón y acero, no apareció hasta bien entrado siglo XX. El hecho de recubrir los perfiles metálicos de los pilares de hormigón en los edificios altos de Estados Unidos, respondía únicamente a una necesidad estricta de protección contra incendio. Edificios como el Seagram de Mies en Nueva York no contemplan el hormigón más que por una simple cuestión de recubrimiento. En el año 1969, Fazlur R.Kahn de SOM propuso por primera vez un edificio en dónde el hormigón de los pilares entraba también en consideración en el cálculo de su resistencia global y no sólo por una cuestión de protección. La aparición del concepto "sección mixta" en edificación -más aún pensando en el caso de pilares para grandes cargas- es de una importancia parecida a la que supuso la aparición del hormigón armado a finales de siglo XIX.

No podríamos concebir la gran mayoría de los edificios altos que se han construido en las últimas décadas sin la posibilidad de plantear estructuras mixtas en forjados, pero especialmente en columnas. El hormigón proporciona al acero unas potencialidades excepcionales de absorción de energía y de protección, que serían difíciles de conseguir con una estructura metálica estricta. El concepto de columna mixta ha ido evolucionando desde un simple perfil en $\mathrm{H}$ embebido en una sección de hormigón, hasta las super-columnas "armadas" con perfiles enormes del Banco de China de I.M. Pei en Hong Kong. Su uso se ha extendido ampliamente como consecuencia de la necesidad creciente de construcción en altura en zonas de fuerte peligrosidad sísmica. En estos casos, la capacidad de absorber energía acompañada de una generosa ductilidad junto con una elevada resistencia a compresión, son cualidades más que necesarias.

\section{SECCIONES TUBULARES MIXTAS}

Lo que en principio fue un proceso normal de aceptación en el caso de secciones mixtas con perfiles embebidos en $\mathrm{H}$, no lo fue para las secciones mixtas tubulares. El desconocimiento y los interrogantes que plantean este tipo de secciones -algunos de los cuales hasta día de hoy-, por disponer de un núcleo macizo de hormigón (a veces en masa sin armar) aplazaron el uso masivo de este tipo de estructuras hasta muy entrado siglo XX. AG Tarics of Reid \& Tarics Associates diseñaron por primera vez en 1972 un edificio entero a base de pilares tubulares mixtos en el centro de San Francisco, el Two Union Square Building, sin conocer aún al cien por cien sus increíbles posibilidades. A lo largo de los años siguientes se ha demostrado que la intuición de AG Tarics estaba en lo cierto para construcciones en zonas de altos índices de sismicidad: los núcleos de hormigón confinados por los perfiles tubulares metálicos poseen una capacidad máxima de absorción de energía, en este caso energía procedente de solicitaciones sísmicas, y constituyen así un exponente muy importante en el diseño de estructuras antisísmicas.

Las posibilidades de esta joven tipología son muchas, y aún no han estado muy explotadas. El hecho de combinar ambos materiales [condición que cumplen en general todas las mixtas] no sólo permite aquí aprovechar sendas cualidades, sino que además las mejora notablemente. El hecho de rellenar los perfiles tubulares de hormigón convierte esta solución en una simbiosis perfecta que maximiza el camino iniciado en la optimización estructural.

Desde la propuesta del Two Union Square Building, la mayoría de normativas por todo el mundo han incorporado este tipo de secciones en sus páginas, proponiendo distintos métodos de análisis y diseño (2) (3). Las primeras fueron las $\mathrm{ACl}$ americanas por cuestión de origen, pero rápidamente muchas otras lo fueron incorporando de una forma u otra, fundamentándose básicamente en las investigaciones que se habían llevado a cabo en las últimas tres décadas del siglo XX sobre este tema.

Tanta es la complejidad de comportamiento de estas secciones, y tantas también sus cualidades, que existen abiertas multitud de líneas de investigación preocupadas por su análisis. Los japoneses, después de los americanos, constituyen una de las escuelas más implicadas en su aplicación por razones evidentes de sismicidad de la región.

\section{EL TUBO COMO SECCIÓN ÓPTIMA}

El avance y la generalización del perfil tubular en la ingeniería civil, pero también en la arquitectura, es debido como se ha comentado, a sus excelentes prestaciones mecánicas. 
3. Gráfico comparativo coeficiente $m$

4. Gráfico comparativo coeficiente $n$
A nivel de rendimiento seccional, el tubo dispone de muy pocos competidores. La sección tubular es la que mejor trabaja a carga axil en columnas esbeltas y también a torsión, dando además una muy buena respuesta a flexión. Estas propiedades, sumadas a su comportamiento por igual en todos sus ejes [resultado de su simetría], constituyen el principal motivo por el que ciertamente cuesta mucho encontrarle competidor.

En las tres tablas siguientes (Tablas 1, 2 y 3) pueden compararse las propiedades mecánicas de distintos tipos de secciones, agrupados por valores de área muy parecidos. Se comparan aquí las respuestas a flexión en los dos ejes (Momentos de Inercia y Momentos resistentes plásticos) y las respuestas a compresión (Radio de giro) entre distintas geometrías seccionales. Puede apreciarse ya a simple vista el excelente rendimiento del tubo en términos generales frente todas las otras:

Para poder realizar un análisis comparativo visual, se han definido dos parámetros teóricos de rendimiento mecánico, $m$ y $n$. Estos dos valores describen la respuesta combinada (en los dos ejes) de las distintas secciones

Tabla 1. Comparación de prestaciones mecánicas en secciones de área aproximada de $5300 \mathrm{~mm}^{2}$.**

\begin{tabular}{|l|l|l|l|l|l|}
\cline { 2 - 6 } \multicolumn{1}{c|}{} & Tubo D (219,8) & HEB 160 & HEM 100 & HEA 200 & IPN 260 \\
\hline Área [x10e2] & 53,1 & 54,3 & 53,2 & 53,8 & 53,3 \\
\hline Inercia Y [x10e4] & 2960 & 2492 & 1143 & 3692 & 5740 \\
\hline Inercia Z [x10e4] & 2960 & 889 & 399 & 1336 & 288 \\
\hline M. Res. Y [x10e3] & 270 & 311 & 190 & 388 & 442 \\
\hline M. Res. Z [x10e3] & 270 & 111 & 75 & 133 & 51 \\
\hline Radio de giro Y & 74,7 & 67,8 & 46,3 & 82,8 & 104,0 \\
\hline Radio de giro Z & 74,7 & 40,5 & 27,4 & 49,8 & 23,2 \\
\hline
\end{tabular}

Tabla 2. Comparación de prestaciones mecánicas en secciones de área aproximada de $10000 \mathrm{~mm}^{2}$.**

\begin{tabular}{|l|l|l|l|l|l|}
\cline { 2 - 6 } \multicolumn{1}{c|}{} & Tubo (323,10) & HEB 280 & HEM 160 & HEA 280 & IPN 360 \\
\hline Área [x10e2] & 98,6 & 103,1 & 97,1 & 97,3 & 97,0 \\
\hline Inercia Y [x10e4] & 12158 & 19272 & 5098 & 13675 & 19610 \\
\hline Inercia Z [x10e4] & 12158 & 6595 & 1759 & 4763 & 818 \\
\hline M. Res. Y [x10e3] & 751 & 1376 & 566 & 1012 & 1098 \\
\hline M. Res. Z [x10e3] & 751 & 471 & 212 & 340 & 114 \\
\hline Radio de giro Y & 111,0 & 121,1 & 72,5 & 118,6 & 142,2 \\
\hline Radio de giro Z & 111,0 & 70,8 & 42,6 & 70,0 & 29,0 \\
\hline
\end{tabular}

Tabla 3. Comparación de prestaciones mecánicas en perfiles de área aproximada de $20000 \mathrm{~mm}^{2}$. **

\begin{tabular}{|l|l|l|l|l|l|}
\cline { 2 - 6 } \multicolumn{1}{c|}{} & Tubo (508,12) & HEB 400 & HEM 240 & HEA 500 & IPN 550 \\
\hline Área [x10e2] & 195,0 & 197,8 & 199,6 & 197,5 & 212,0 \\
\hline Inercia Y [x10e4] & 59755 & 57684 & 24290 & 86980 & 99180 \\
\hline Inercia Z [x10e4] & 59755 & 10819 & 8153 & 10367 & 3490 \\
\hline M. Res. Y [x10e3] & 2353 & 2884 & 1799 & 3550 & 3606 \\
\hline M. Res. Z [x10e3] & 2353 & 721 & 657 & 691 & 349 \\
\hline Radio de giro Y & 175,0 & 170,8 & 110,3 & 209,8 & 216,3 \\
\hline Radio de giro Z & 175,0 & 74,0 & 63,9 & 72,4 & 40,6 \\
\hline
\end{tabular}

** Diámetro en mm, Área en $\mathrm{mm}^{2}$, Inercia en $\mathrm{mm}^{4}$, Momento Resistente en $\mathrm{mm}^{3}$ y Radio de Giro en $\mathrm{mm}$, según el Sistema Internacional de unidades (4). en función del área. Vienen definidas por las siguientes expresiones [1] y [2]:

$$
\begin{aligned}
& \mathrm{m}=\frac{\left(\mathrm{I}_{\mathrm{y}}+\mathrm{I}_{\mathrm{z}}\right)}{2 * \mathrm{~A}} \\
& \mathrm{n}=\frac{\left(\mathrm{i}_{\mathrm{y}}+\mathrm{i}_{\mathrm{z}}\right)}{2 * \mathrm{~A}}
\end{aligned}
$$

Siendo $I_{y}$ y $I_{z}$ los momentos de inercia, $i_{y} y$ $\mathrm{i}_{2}$ los radios de giro y $\mathrm{A}$ el área de la sección (como valor indicador de rendimiento material); por lo que los parámetros $m$ y $n$ describen con precisión la respuesta global de las secciones, más allá de cada uno de los parámetros resistentes aislados.

En los dos gráficos siguientes (Figuras 3 y 4), se hace explícito como para distintos rangos de cantidad de material (áreas en el eje de abscisas), ambos coeficientes en el caso de secciones tubulares se mantienen por encima de los correspondientes para el resto de tipologías (eje de ordenadas). A mayores valores de $m$ o $n$, mayor es el rendimiento de las secciones, a efectos de una respuesta combinada en las dos direcciones.

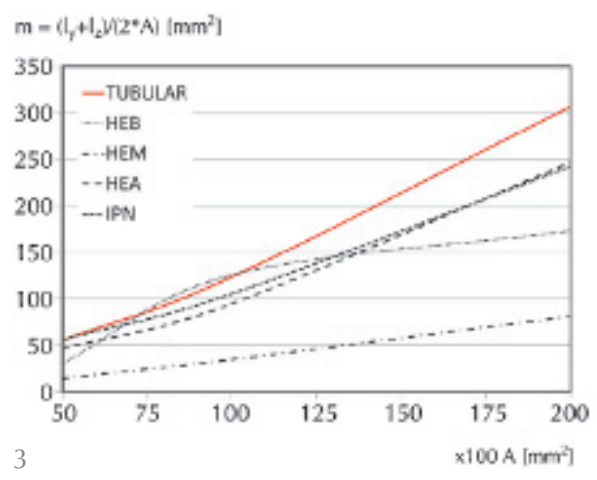

$\left.\mathrm{n}=\left\langle\mathrm{i}_{4}+\mathrm{i}_{2}\right\rangle \mid 2 * \boldsymbol{A}\right\}$

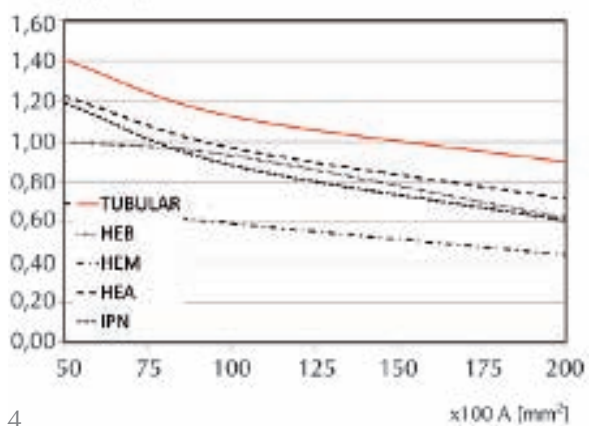

Estas excelentes prestaciones frente a todo tipo de solicitaciones quedan explicadas más detenidamente en los apartados siguientes, así como las expresiones que permiten obtener sus parámetros mecánicos.

\section{Respuesta a carga axil}

La capacidad de una sección a resistir compresiones no sólo depende de la resisten- 
cia propia del material que la compone, sino fundamentalmente de su capacidad resistente a pandeo. Esta cualidad depende básicamente de la esbeltez de la barra, y el parámetro geométrico que mejor puede definir este comportamiento es el del radio de giro, definido por la expresión [3]:

$$
i_{x}=\sqrt{\frac{I_{x}}{A}}
$$

Es decir, la raíz cuadrada de la relación inversa entre cantidad de material (área) y como de alejado del centro de gravedad está dispuesto este material (inercia). Así, a mayor radio de giro mayor resistencia opondrá la sección a pandear y más capacidad de carga tendrá la sección a compresión.

Una sección puede tener mucha inercia y a la vez poco radio de giro en un determinado sentido, si esta inercia va acompañada de un área también importante. En este sentido, la sección tubular no tiene literalmente competidor, porque además es simétrica en los dos ejes y responde por igual a pandeo en ambas direcciones.

Los Eurocódigos (2) introducen un nuevo parámetro para definir la esbeltez de una barra: la esbeltez reducida. Ésta se define como la raíz cuadrada de la relación entre la resistencia plástica de una sección y su carga crítica de Euler, según la expresión [4].

$$
\bar{\lambda}=\sqrt{\frac{N_{p l, R}}{N_{c r}}}
$$

Siendo la carga crítica de Euler $N_{c r}$, aqueIla carga máxima por la que un pilar entra de forma teórica en inestabilidad por pandeo (en el caso de suponer que este axil sea completamente centrado) y $N_{p l, R d}$ la carga plástica de dicha sección (es decir, la respuesta resistente máxima de ambos componentes). La expresión para hallar la carga crítica viene definida por la teoría elástica: [5]

$$
N_{c r}=\frac{\pi^{2} E I}{L_{k}{ }^{2}}
$$

\section{Respuesta a torsión}

Las secciones que mejor trabajan a torsión son aquéllas de geometría cerrada, porque son las menos sensibles a deformación rotacional; el máximo exponente de las secciones cerradas lo constituyen evidentemente las tubulares, por lo general. La inercia a torsión de un perfil tubular puede calcularse fácilmente mediante la expresión [6].

[6]

$$
I_{T}=\frac{\pi}{2} \cdot\left(R^{4}-r^{4}\right)
$$

Evidentemente, una sección maciza tendría mejor respuesta absoluta a torsión que una tubular, pero también con mucho más área y por tanto, mucho más desperdicio de material. Si se compara en términos de rendimiento material, el tubo vuelve a destacar sobre las otras secciones por su extraordinaria eficacia. $Y$ es que todos los perfiles abiertos en $\mathrm{T}$ o en $\mathrm{H}$ ofrecen una respuesta realmente nefasta a solicitaciones de torsión, y las normas de aplicación son muy restrictivas en estos casos.

Ya en la primera década del siglo XX, L. Prandlt explicó la distribución tensional en secciones sometidas a torsión utilizando la conocida analogía de la membrana. Este principio relaciona elocuentemente la ecuación diferencial que define las tensiones en cada punto de una sección a torsión, con la deformación que experimentaría una membrana fijada en el perímetro de dicha sección y bajo una presión uniforme.

Determinados problemas matemáticos pueden interpretarse fácilmente de forma intuitiva, y el comportamiento tensional de una sección sometida a torsión es un claro ejemplo de ello, como demuestran fehacientemente Galeano y Ramírez en su investigación (7). Se entiende que cualquier sección cerrada tiene una mejor respuesta a torsión, en comparación con otra abierta, a igualdad de área.

El hecho de responder muy bien a torsión conlleva que las secciones tubulares sean las más idóneas para una concepción tridimensional de las estructuras. El momento torsor constituye la tercera dimensión "desconocida" y muchas veces desestimada de las arquitecturas de pórticos planos que tradicionalmente se han proyectado. Nuevos retos geométricos piden también a las estructuras nuevas secciones más polivalentes.

\section{Respuesta a flexión}

La resistencia a flexión viene fundamentalmente definida por un determinado momento de inercia. El concepto de momento de inercia define perfectamente como está distribuido el material en una sección; a más alejado del centro de gravedad, mayor será su valor. Cada eje de la sección tendrá su correspondiente momento de inercia, por lo que su capacidad a flexión dependerá de qué dirección tenga la solicitación.

Analizando este último concepto, el tubo concentra toda su área justo en su perímetro, alejándose el máximo posible del c.d.G. A pesar de ello, cierto es que la flexión debe resistirse en una determinada dirección, y si bien el tubo presenta su máxi- 
5. Proceso de optimización seccional.

6. Decrecimiento de resistencia a compresión ma inercia en todas direcciones por igual, existen determinadas secciones en $\mathrm{H}$ o I que para una misma cantidad de material superan este último en una dirección concreta, pero nunca en la combinada.

El momento de inercia a flexión de un tubo puede determinarse, de forma semejante a la inercia a torsión (6), a partir de la expresión [7].

$$
I_{F}=\frac{\pi}{4} \cdot\left(R^{4}-r^{4}\right)
$$

Y su módulo resistente plástico como:

$$
W_{p l}=\frac{4}{3} \cdot\left(R^{3}-r^{3}\right)
$$

La principal ventaja de las secciones tubulares a flexión es por lo tanto, su respuesta por igual sea cual sea la dirección del momento, hecho que las convierte en extremadamente versátiles en una arquitectura que cada día es de concepción más global.

\section{Respuesta a cortante}

Su comportamiento a cortante es también mejor que el de una sección abierta en $\mathrm{H}$, en términos de igualdad de área. El cortante es un esfuerzo resistido por todas aquellas pletinas que componen el alma del perfil; en el caso de un perfil tubular, todo su perímetro queda solicitado dando por consiguiente una muy buena respuesta a los esfuerzos tangenciales.

El cortante que resiste una sección tubular puede calcularse a partir de la expresión siguiente [9], según (7):

$$
V_{p l, R d}=\frac{2 \cdot A}{\pi} \cdot \frac{f_{y d}}{\sqrt{3}}
$$

Obviamente, existen unos límites referidos a esbelteces de chapas, en relación a las abolladuras locales. Estos límites son de aplicación en todos los casos anteriores, y en todo caso están muy bien definidas en la literatura específica (8).

\section{NACIMIENTO DE UNA NUEVA SECCIÓN}

El último gran avance en el ámbito de las secciones mixtas tubulares lo han realizado unos ingenieros lituanos desde la Universidad Gediminas Technical University de Vílnius, a través de una nueva sección, fruto de someter las tubulares mixtas a un último proceso de optimización geométrica, en un momento en el que parecía que la vía seccional estaba ya agotada.
La nueva hipótesis de optimización se fundamenta en la propuesta de unas secciones mixtas tubulares aligeradas en el núcleo, pensadas expresamente para construirse prefabricadas; es decir, formadas a partir de un vaciado parcial de los núcleos de las secciones tubulares Ilenas de hormigón. De este modo, y según la ecuación [3], al reducir el hormigón del núcleo, la proporción entre inercia y área de la sección aumenta porque se elimina de ésta el material justo en aquellas zonas más próximas al centro de masas $-y$ por consiguiente, menos eficaces.

Los tubos metálicos presentan un mejor comportamiento relativo a pandeo, mientras que las secciones mixtas tubulares tienen una mejor resistencia a compresión. La nueva sección propuesta por Kuranovas y Kvedaras (9) (10) sintetiza los beneficios de ambas para proporcionar así una sección optimizada (Figura5). El hormigón ya no se plantea como núcleo macizo interior, sino sólo como capa de recubrimiento de la cara interior del perfil tubular, que trabaja gracias al contacto existente entre ambas superficies (9) (11).

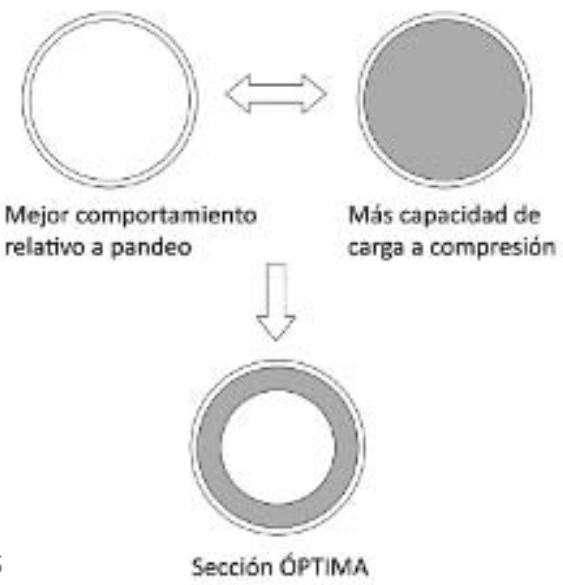

Analizando el comportamiento a pandeo de una sección tubular vacía y comparándolo con el de una sección tubular llena de hormigón, puede observarse rápidamente que a medida que se incrementa la altura del pilar, disminuye proporcionalmente más la capacidad de carga a compresión en el perfil lleno que en el vacío, (Figura 6).

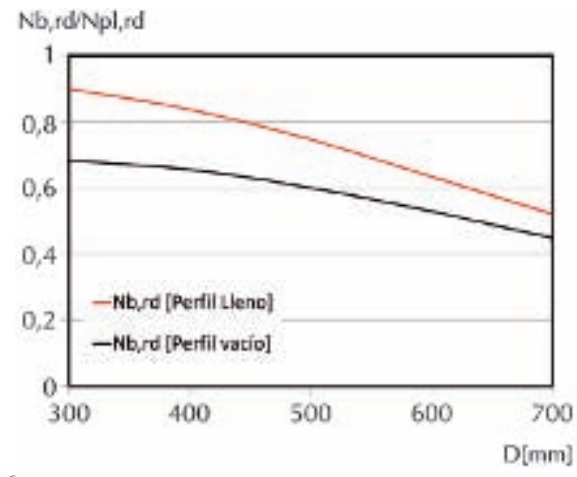


Así, para cada sección tubular -mixta o noexiste un rango de alturas y de proporción de grosores óptimo para columnas esbeltas (Figura 7) que le permite aprovechar al máximo las prestaciones de los materiales, considerando también los efectos de pandeo. Esta relación óptima nos confirma la presencia de muchas de las formas que encontramos en la naturaleza como ejercicio ejemplar de sostenibilidad material. La propuesta de los lituanos no es del todo inédita, como en la mayoría de hallazgos formales del hombre; si buscamos en la naturaleza, encontramos multitud de elementos que reproducen las secciones aligeradas en capas de Kuranovas y Kvedaras (12): por ejemplo, una sección del fémur humano (Figura 8).

Comparando la máxima resistencia plástica de la sección $N_{p l R d}$ con su carga crítica de Euler $N_{c r}$ para un determinado rango de longitudes de pilar (cada sección pedirá un análisis en un intervalo diferente de alturas), podemos observar que existe un punto donde se cruzan las dos funciones. Allá donde la carga crítica valga lo mismo que la resistencia plástica, estaremos optimizando el área de la sección. En la (Figura 7) se representa este punto para un ejemplo de sección tubular de $219.1 \mathrm{~mm}$ de diámetro y $3 \mathrm{~mm}$ de espesor, rellena de hormigón de 25MPa y de acero S275 -según prontuario de ARCELOR@ (4). Puede verse que la optimización máxima de esta sección en cuestión se produce para unas alturas de entre 6 y 7 metros, intervalo en el que el grosor de hormigón interior óptimo variará entre 0 y el radio interior.

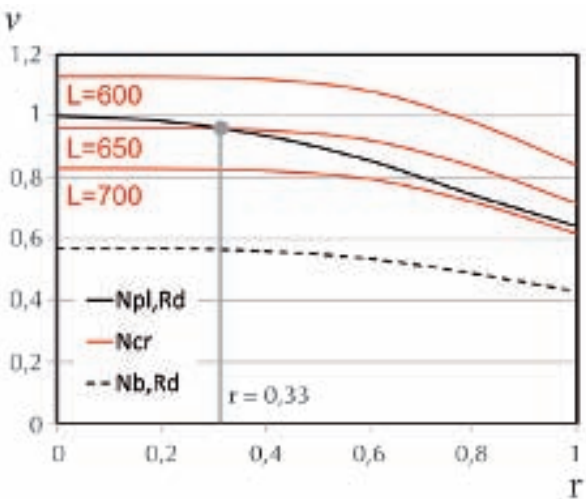

Siendo el eje de ordenadas el axil adimensional $v$, definido por: [10]

$$
v=\frac{\mathrm{N}}{\mathrm{N}_{\mathrm{pl}, \mathrm{Rd}}}
$$

y las abscisas la proporción de vacío sobre lleno r, definida por la siguiente expresión geométrica [11], y según la (Figura 9).

$$
r=\frac{E_{0}}{E_{0}+E_{c}}
$$
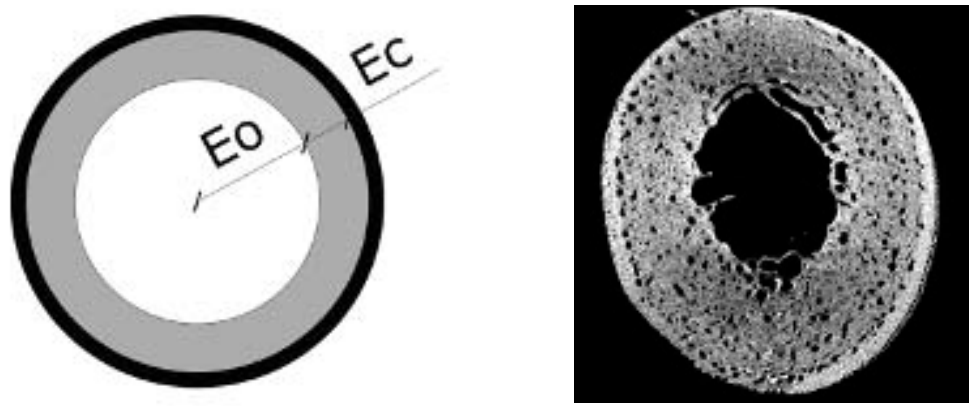

Siguiendo el proceso anterior para un rango distinto de diámetros y longitudes de barra, se pueden obtener una colección de superficies de puntos de proporciones óptimas, en función del espesor de chapa, a partir de la que el proyectista podría escoger en cada caso cuales son las proporciones entre diámetro y grosor de la capa de hormigón que más le interesan para su carga y altura específicas (Figura 10)

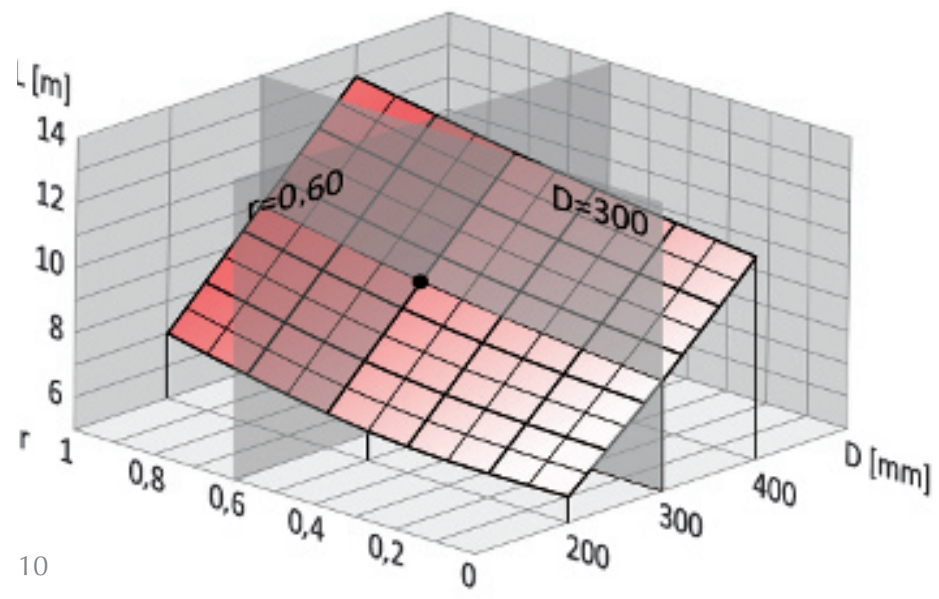

Con el objetivo de agilizar el uso de estas superficies, se propone la obtención de diagramas aislados para cada porcentaje de radio de hueco sobre radio total de la sección. Así, entrando desde un diámetro por las abscisas y conociendo la longitud del pilar en las ordenadas, rápidamente puede obtenerse la proporción de diseño más óptima para columnas esbeltas en cada caso (Figura 11). Evidentemente, este criterio no tiene en cuenta la carga, sino solamente un aprovechamiento máximo del material frente a maximización de carga y esbeltez, por lo que resulta útil para comprobar lo distanciado que puede estar un diseño concreto de lo que sería una columna ideal. Es evidente que la proporción óptima no es la que mayor carga resiste, sino la que ofrece el máximo rendimiento con la máxima altura posible, y sólo para columnas esbeltas.

\section{EN REFERENCIA A LA EJECUCIÓN Y PUESTA EN OBRA}

Las secciones tubulares aligeradas se plantean desde un inicio con el propósito de extrapolarlas en el campo de la prefabrica-
10. Diagrama de proporciones óptimas para perfiles tubulares de $4 \mathrm{~mm}$ de espesor.

11. Curvas de proporción óptima para secciones tubulares de chapas de $4 \mathrm{~mm}$ de espesor.

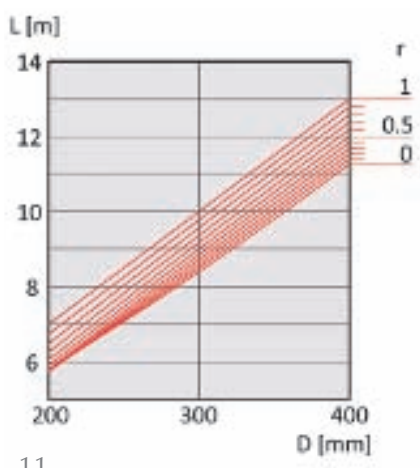


12. Imágenes de muestras obtenidas por centrifugado (10).

13. Máquina de centrifugado Montaje en obra. Uniones.

14. Vista general de un nudo. ción industrial. Es decir, además de la optimización que representan a nivel geométri$\mathrm{CO}$, las secciones mixtas aligeradas pueden favorecer un paso más allá en la construcción con prefabricación en taller y montaje en obra. Las ventajas de evitar el hormigonado in situ son muchas, desde la adherencia hasta el control de calidad.

\section{Prefabricación de las secciones.}

El método que propone la escuela lituana para la prefabricación de este tipo de secciones es el de centrifugado (10): el hormigonado de las secciones a partir de la rotación controlada. El proceso, a pesar de parecer utópico, presenta multitud de ventajas e incluso puede llegar a mejorar la capacidad resistente de la sección, a base de la compactación que ejerce la aceleración centrípeta del perfil dentro la centrifugadora.

El proceso para la fabricación de las secciones pasa por introducir la masa de hormigón en el interior del tubo y centrifugar la mezcla a una determinada velocidad angular, hasta que quede un grosor deseado de la primera capa; así va realizándose hasta obtener distintas capas con distintos grosores (Figura 12). Además, el hecho de fabricar la sección en multicapa favorece activamente su capacidad resistente, tal y como han demostrado estos autores. La máquina de centrifugado planteada por Kuranovas (12) tiene el aspecto de la (Figura 13).

De esta forma la masa de hormigón -de árido muy fino- va repartiéndose homogéneamente por el perímetro interior del tubo
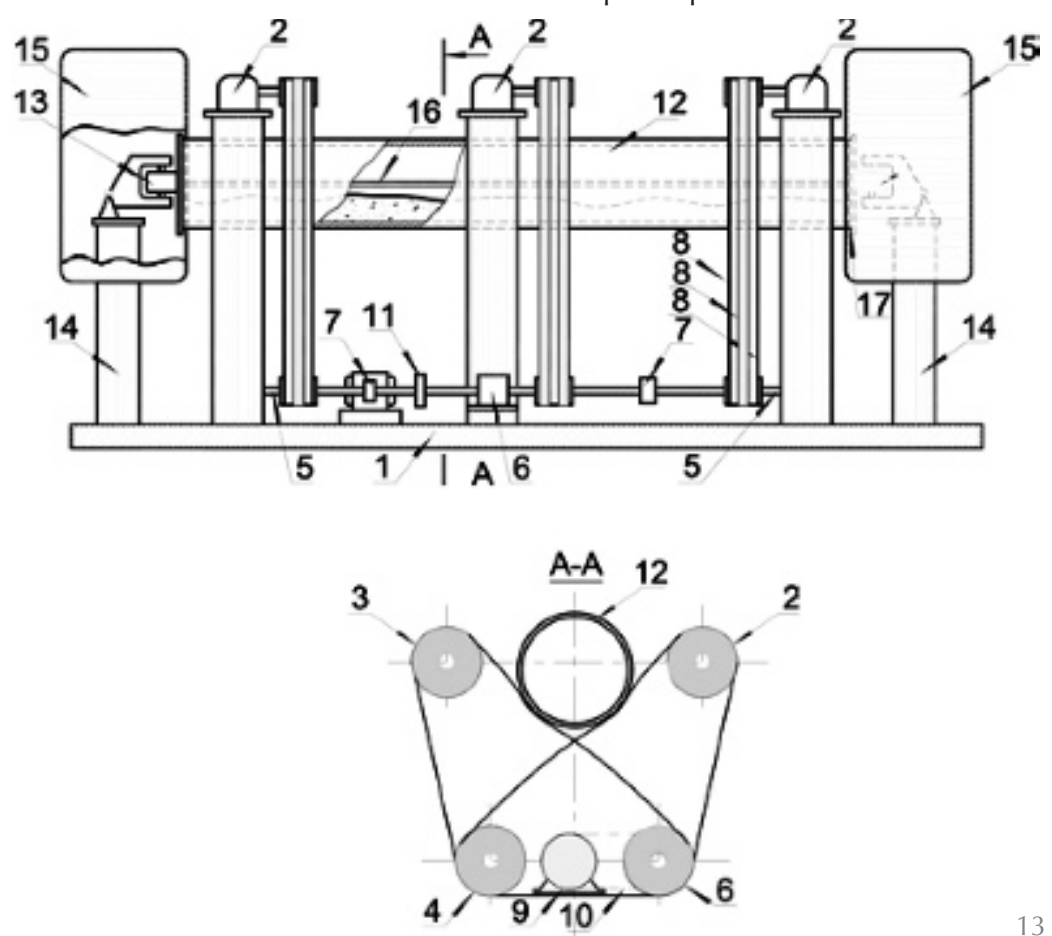

de acero y compactándose en función del grosor de la capa y de su velocidad angular. La compactación se produce a base de expulsar el agua contenida en la mezcla como consecuencia de la presión a que está sometida a través de micro-canales que se forman en el propio material.

La ejecución de este tipo de secciones pretende ser sencilla, rápida y eficiente, reduciendo al máximo las deficiencias en obra y maximizando el rendimiento a partir de la optimización específica para cada problema concreto. A partir de este proceso de fabricación industrializado en taller puede controlarse muy bien cuál es la resistencia final de la sección, y posiblemente reducir los coeficientes de seguridad generales.

Al ser secciones mixtas prefabricadas y pensadas para construirse en obra completamente en seco, las uniones pueden resultar un tanto complicadas si no aparece este tema en la misma fase de diseño. El objetivo es reducir al máximo las operaciones en obra, así como hormigonados in situ de rellenos o juntas. Este artículo propone un sistema de resolución de las uniones por ensamblaje, sin perforar los perfiles tubulares -ya que las capas interiores de hormigón quedarían dañadas- y minimizando al máximo soldaduras decisivas en obra.

La solución propuesta se fundamenta en un ensamblaje de los dos pilares concurrentes en un nudo entre ellos a cota de forjado, a partir de unos anillos de transmisión a los que se les ha soldado previamente en taller las típicas pletinas de conexión (Figura14). Éstas se usan ya habitualmente en estructuras de pilares mixtos tubulares y consiguen convertir los cortantes de las jácenas en axiles a los pilares, pero no transmitir los momentos. Se trata, por consiguiente, de uniones articuladas, por otra parte normales en estructuras prefabricadas; esto significa que la rigidez transversal del edificio deberá conseguirse mediante algún otro sistema independiente.

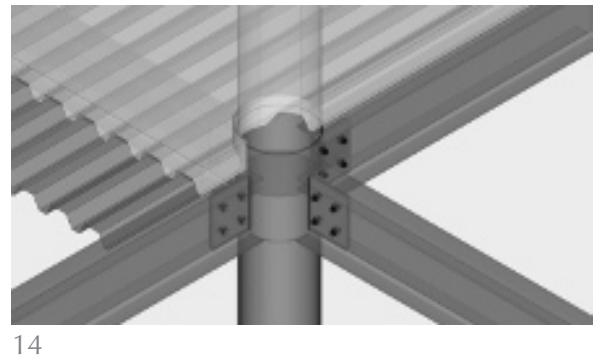

La unión se plantea a través de un anillo metálico -soldado en taller- que encajará perfectamente con el diámetro del tubo inferior. Dicho anillo debería disponer también ya soldadas unas pletinas en las 
direcciones en las que posteriormente se atornillarán las jácenas. La longitud de este anillo será la suficiente para garantizar la perfecta transmisión de compresiones (Figura 15).

De un modo parecido se resolverán las placas base de anclaje en cimentación, en las que el anillo metálico quedará soldado mediante rigidizadores. De este modo, la construcción del esqueleto estructural prefabricado puede realizarse enteramente en seco.

\section{APLICABILIDAD. CONCLUSIONES}

Esta nueva aportación tipológica, pero también metodológica, planteará un nuevo reto en la industria de la construcción, en especial la construcción rápida en altura, high-rise. En los momentos de profundos cambios que se están viviendo y de estricta rentabilización de la arquitectura de los últimos años, no es de extrañar que esta nueva opción pueda convertirse en una seria alternativa a la construcción mixta tradicional.

El camino hacia la prefabricación es sin duda cada vez más imparable por cuestiones evidentes de calidad, tiempo y coste; cualquier aportación en el mundo de los prefabricados es aire fresco para un mundo que necesita reestructurarse y reinventar sus metodologías. El ámbito en que las secciones mixtas tubulares aligeradas pueden tener más interés es sin duda en el de la construcción secuencial en altura, ya que la posibilidad de construir acero y hormigón en una sola fase reduciría de forma importante los problemas derivados de las fases de ejecución, Liew y Xiong (12) (13).

El mejor argumento para su aplicabilidad es el de la mejora sustancial de su rendimiento, a base de una reducción notable de material y un aumento relativo de la resistencia a pandeo; cabe destacar también la reducción de coste que supone la rapidez de ejecución. Además, tal y como se ha comentado anteriormente, una fabricación industrializada proporciona muchas más garantías que una construcción in situ y por lo tanto, esta nueva metodología permitiría plantearse una reducción en los coeficientes de minoración del hormigón y en los coeficientes de seguridad de ejecución generales.

El ahorro en el uso de estas secciones procede de múltiples factores: a) Un aumento

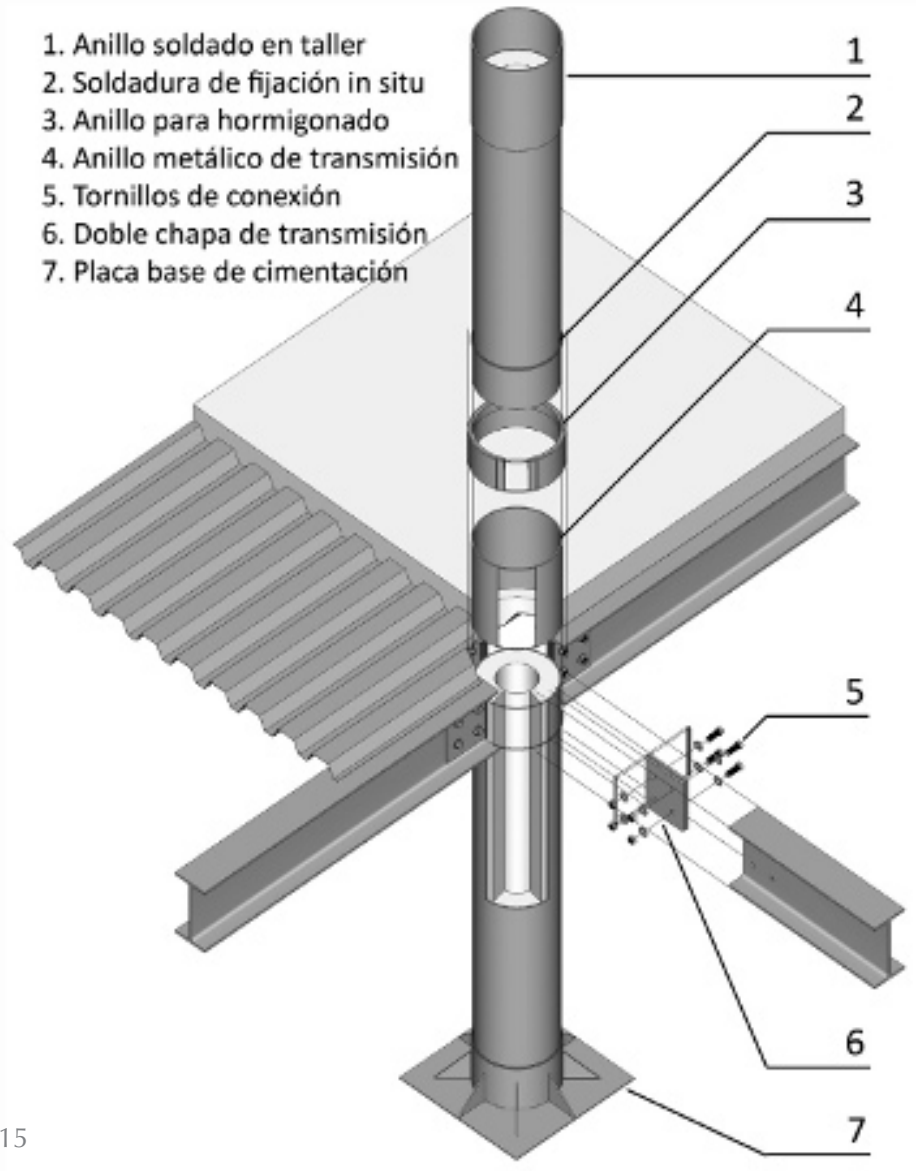

relativo de la capacidad a pandeo, por lo tanto, más resistencia de la sección; b) Una disminución de la cantidad de material; c) Una posible reducción de los coeficientes de seguridad; d) Una mayor rapidez de ejecución; y e) Una reducción de todos aquellos efectos parásitos de la construcción secuencial.

A pesar de todo, cabe subrayar que la construcción prefabricada con este tipo de secciones conllevaría nuevos retos a nivel de análisis global, ya que la resolución de las uniones entre plantas que se propone es articulada y precisa de la resolución de la rigidez transversal a través de otros elementos. Retos a los que en muchos casos están planteándose soluciones reales, ya que muchas de las estructuras construidas con pilares tubulares mixtos ya consideran los nodos como articulados, por la dificultad de rigidizarlos.

En base a los criterios y soluciones iniciadas en este artículo, podríamos empezar a hablar de construcción prefabricada mixta¿si existe de hormigón, por qué no como combinación de dos materiales? 


\section{REFERENCIAS}

(1) Margarit, J., Buxadé, C. (1972). Las mallas espaciales en arquitectura, Barcelona. Ed. Gustavi Gili.

(2) EUROCODE 4. (2004). Design of composite steel and concrete structures, Part 1.1: General rules and rules for buildings. EN 1994-1-1:2004. Brussels (Belgium): European Committee for Standardization.

(3) ANSI/AISC 360-10. (2010). Specification for Structural Steel Buildings. American Institute of Steel Construction, AISC. Segunda Edición.

(4) Catálogo ARCELOR@ UAHE. (2001) Unión de Almacenistas de Hierros de España, Madrid.

(5) Galeano, C., Ramírez D.A. (2003). Aplicación experimental del método de la analogía de la membrana en la determinación de los esfuerzos generados en la torsión de barras de sección no circular. Scientia et technica, (21).

(6) Corus Tubes (2002). Design guide for SHS concrete filled columns. Ed. CorusTubes.

(7) Moga, P., Pacurar, V. (2006). Circular Composite Columns. Mechanism of Shear Transfer. Ovidius University Annals Series: Civil Engineering. 1(8): 27-30.

(8) Hatzigeorgiou, G. D. (2007). Numerical model for the behaviour and capacity of circular CFT columns. Part I and II. Engineering Structures, (30):1579-1589.

(9) Kuranovas, A. Kvedaras, A.K. (2007). Behaviour of hollow-concrete steel tubular composite elements. Journal of Civil Engineering and Management, 13(2): 131-141.

(10) Kuranovas, A., Kvedaras, A.K. (2007). Centrifugally manufactured hollow concrete-filled steel tubular columns. Journal of Civil Engineering and Management, 13(4): 297-306.

(11) Partaukas, N., Bareisis, J. (2008). The stress state in two-layer hollow cylindrical bars. Revista Kaunas University of Technology, 1(75): 5-12.

(12) Liew, R., Xiong, D.X. (2009). Effect of preload on the axial capacity of concrete-filled composite columns. Journal of Constructional Steel Research, (65): 709-722.

(13) Han, L.; Yao, G. (2003). Behaviour of concrete-filled hollow structural steel (HSS) columns with pre-load on the steel tubes. Journal of Constructional Steel Research, (59): $1455-1475$ 